\title{
A EDUCAÇÃO NO DISCURSO DE UMA EQUIPE DE SAÚDE DA FAMÍLIA
}

\author{
Marina da Silva Sanes ${ }^{1}$, Tatiane Alonso Arrieche², Maria Elizabeth Carvalho Cestari ${ }^{3}$
}

\begin{abstract}
RESUMO: Este estudo, de abordagem qualitativa e de tipo descritivo, teve por objetivo identificar o discurso referente à educação no ambiente de trabalho, expresso por membros de uma equipe multiprofissional da Saúde da Família no Município de Rio Grande, Estado do Rio Grande do Sul. A coleta de dados ocorreu de março a junho de 2009, com a utilização de entrevistas não estruturadas a nove profissionais. O corpus do trabalho foi analisado conforme a análise de discurso, utilizando-se a paráfrase e a polissemia como dispositivos analíticos. Identifica-se no discurso predomínio da concepção de educação tradicional, considerando que os profissionais referiram-na como um ato pontual, exterior e de cunho individual; entende-se a educação como construída entre os sujeitos. A análise permitiu a identificação dos discursos presentes na equipe e possibilitou sua compreensão numa estrutura maior de sentidos, instituídos pela educação em geral, e utilizados na formação de profissionais da saúde.
\end{abstract}

PALAVRAS-CHAVE: Educação; Saúde da família; Recursos humanos em saúde.

\section{EDUCATION IN THE DISCOURSE OF A FAMILY HEALTH TEAM}

\begin{abstract}
This study adopted a qualitative and descriptive approach, and it aimed to identify the discourse relating to education in the workplace, expressed by members of a multidisciplinary team of Family Health in the municipality of Rio Grande, Rio Grande do Sul . Data collection occurred from March to June 2009, with the use of unstructured interviews to nine professionals. The corpus of this study was analyzed according to the discourse analysis technique, using paraphrase and polysemy as analytical devices. A prevailing notion of traditional education, whereas the professionals mentioned it as an isolated act, outdoor and on an individual level is identified in the discourse, meaning that education is constructed among subjects. The analysis allowed the identification of discourses present within the team and allowed its understanding from a larger structure of senses, set up by education in general, and used in the training of health professionals.
\end{abstract}

KEYWORDS: Education; Family health; Health human resources.

\section{LA EDUCACIÓN EN DISCURSO DE UN EQUIPO DE SALUD DE LA FAMILIA}

RESUMEN: Este estudio, de abordaje cualitativo y de tipo descriptivo, tuvo por objetivo identificar el discurso referente a la educación en ambiente de trabajo, expresado por miembros de un equipo multiprofesional de la Salud de la Familia en municipio de Rio Grande, estado de Rio Grande do Sul. Los datos fueron recogidos de marzo a junio de 2009, con la utilización de entrevistas no estructuradas a nueve profesionales. El corpus del trabajo fue analizado de acuerdo con el análisis de discurso, utilizándose la paráfrasis y la polisemia como dispositivos analíticos. Se identifica, en discurso, predominio de la concepción de educación tradicional, considerando que los profesionales hablaron de ella como un acto puntual, exterior e individual; se entiende la educación como construida entre los sujetos. El análisis permitió la identificación de los discursos presentes en equipo y posibilitó su comprensión en una estructura mayor de sentidos, instituidos por la educación en general, y utilizados en la formación de profesionales de la salud.

PALABRAS CLAVE: Educación; Salud de la familia; Recursos humanos en salud.

${ }^{1}$ Enfermeira. Mestre em Enfermagem. Residente em Saúde da Família e Comunidade do Grupo Hospitalar Conceição de Porto Alegre-RS.

${ }^{2}$ Enfermeira. Mestre em Enfermagem. Enfermeira do Hospital Universitário Miguel Riet Correa Júnior, da Universidade Federal do Rio Grande-HU-FURG.

${ }^{3}$ Enfermeira. Doutora em Enfermagem. Professora Associada do Departamento de Enfermagem da FURG.

Autor correspondente:

Maria Elizabeth Carvalho Cestari

Universidade Federal do Rio Grande

Rua General Osório, sn - 96201-900 - Rio Grande-RS, Brasil

E-mail: bethcestari@yahoo.com.br

Recebido: 01/07/09

Aprovado: 05/08/10

Cogitare Enferm. 2010 Jul/Set; 15(3):480-5 


\section{INTRODUÇÃO}

A análise de discurso, como disciplina e metodologia descrita pela Escola Francesa, e no Brasil evidenciada no trabalho de Orlandi, utiliza-se da busca de sentidos produzidos entre os seres humanos, em um determinado contexto e momento histórico, considerando-se as condições de produção, os protagonistas e o objeto do discurso ${ }^{(1)}$.

Entendida como um ponto de vista do qual pode partir o analista, a análise de discurso permite compreender os modos de funcionamento da linguagem, concebida como "mediação necessária entre o homem e a realidade natural e social. Essa mediação, que é o discurso, torna possível tanto a permanência e a continuidade quanto o deslocamento e a transformação do homem e da realidade em que ele vive"(1:15).

Sendo o discurso o objeto de análise, considera-se que este possui uma característica sóciohistórica, ou seja, não consiste em mera transferência de informações, traduz mais: "é o efeito de sentidos entre sujeitos, não estando no dizer de um interlocutor ou no ouvir de outro; encontra-se no espaço discursivo (intervalo) criado (constituído) pelos dois interlocutores"(1:15).

A análise de discurso, como metodologia utilizada na área da saúde, pode ser uma estratégia tanto no campo da pesquisa em saúde quanto no fazer cotidiano dos profissionais, já que permite ao analista "situar (compreender), e não apenas refletir, o gesto de interpretação do sujeito e expor seus efeitos de sentido"(1:15). De formas distintas e com referenciais variados, estudos têm enfatizado a utilização da análise de discurso aplicada à área da saúde ${ }^{(2-3)}$.

$\mathrm{Na}$ realidade do SUS, especificamente, na Saúde da Família, com a proposta de mudança no modelo assistencial, torna-se interessante compreender os discursos vigentes que cercam os sujeitos e as realidades de atenção à saúde ${ }^{(4)}$. Como reorientar um modelo de atenção senão compreendendo os modos como o discurso hegemônico na saúde funciona e produz sentidos entre profissionais e usuários do SUS para que outro dizer seja possível? A Saúde da Família, nessa perspectiva de reorientação do modelo de atenção, pode se constituir como potencial força geradora de mudanças estruturais, com a inclusão de um viés sanitário-epidemiológico ${ }^{(5)}$.

Uma discussão possível e reconhecida como necessária para a mudança proposta no modelo assistencial compete à educação, aqui discutida através da incorporação dos pressupostos da Educação Permanente em Saúde (EPS), que busca discutir e analisar temáticas da realidade dos trabalhadores, construindo conjuntamente com eles uma nova forma de agir na saúde.

Para que os profissionais de saúde possam transformar as práticas do trabalho em saúde, é necessário que se sintam incomodados ou inquietados; é preciso que algo que esteja instituído demonstre sua incapacidade de manutenção, seja uma prática, uma postura, uma atitude, um dizer, um discurso. É essencial que o instalado que ora incomoda seja discutido, dialogado e pensado em todas as suas dimensões. Neste caso, a educação permanente tem espaços para ser construída ${ }^{(6)}$.

Sabe-se que a educação permanente por si só não transforma as práticas. Porém, o desenrolar deste processo pedagógico pode ser amplamente explorado no sentido de favorecer o crescimento dos sujeitos e possibilitar a mudança, centralizando a educação como fonte propulsora para transformação de homens e mulheres. Desta forma, permite sensibilizar os profissionais na direção das mudanças necessárias para a reorientação do modelo assistencial ${ }^{(7-8)}$.

$\mathrm{Na}$ visão do analista de discurso, surgem alguns questionamentos, como: que concepção de educação atualmente permeia as práticas de atenção à saúde? quais sentidos de educação são produzidos entre os trabalhadores?

O que é importante de ser desvelado é como a educação é construída e entendida por um determinado grupo de pessoas; e, desta forma, compreender ou determinar se os processos educativos que estão sendo desenvolvidos nas instituições de saúde podem, realmente, transformar as práticas profissionais, tal como se propõem.

Objetivou-se, neste estudo, identificar o discurso referente à educação no ambiente de trabalho expresso por membros de uma equipe multiprofissional da Saúde da Família no Município do Rio Grande/RS.

\section{METODOLOGIA}

Este artigo caracteriza-se por uma abordagem qualitativa, do tipo descritivo, com a utilização da análise de discurso. O cenário do estudo compõe-se de uma Unidade Básica de Saúde da Família, localizada na zona oeste do Município de Rio Grande. Os sujeitos da pesquisa fazem parte da equipe multiprofissional de saúde que atua na UBSF, composta por cinco agentes comunitários de saúde, duas auxiliares 
de enfermagem, uma médica e uma enfermeira, sendo que todos aceitaram participar da pesquisa.

A coleta de dados ocorreu no período de março a junho de 2009, com a utilização de entrevistas não-estruturadas, sendo organizadas com a seguinte questão norteadora: existe um processo educativo nesta unidade para os profissionais de saúde? fale sobre isso.

As entrevistas foram gravadas e transcritas na íntegra, constituindo o corpus do material produzido, valendo-se da premissa de que o texto produzido pela transcrição dos materiais não é a finalidade da análise de discurso, mas sim o próprio discurso ${ }^{(1)}$.

O corpus do trabalho foi analisado conforme as seguintes etapas: 1) fase de passagem da superfície linguística para o objeto discursivo, 2) fase de passagem do objeto discursivo para o processo discursivo, 3) fase de passagem do processo discursivo para a formação ideológica.

A primeira etapa consta da de-superficialização do material que será analisado, ou seja, é um avanço na superfície linguística das palavras, sem contextualização; é a forma de passar do texto para o discurso $^{(1)}$. Os dispositivos analíticos utilizados para aprofundamento na compreensão do discurso dos sujeitos foram a paráfrase e a polissemia. A primeira consiste naquilo que se mantém nos discursos dos sujeitos, o que está na memória do dizer, que tem uma materialidade histórica, o que é dito num contínuo de dizeres de diferentes formas. Já a polissemia estabelece a complementaridade: não há paráfrase sem processo polissêmico, de modo que uma tensão entre a continuidade e a ruptura é própria da linguagem ${ }^{(2)}$. A polissemia constitui a ruptura de determinado discurso com sua anterioridade; é o dito que destoa, o diferente, aquilo que intercepta a continuidade da paráfrase $^{(3)}$.

$\mathrm{Na}$ segunda etapa constrói-se o processo discursivo - um nível de interpretação mais profundo - que pretende abarcar as formações discursivas, as quais são responsáveis pela produção de sentidos em determinado contexto (imediato e mediato), ou seja, é o momento de encontrar o que pode e deve ser dito, assim como aquilo que não pode e nem é dito naquele contexto específico ${ }^{(1)}$.

$\mathrm{Na}$ última etapa, são apreendidas as formações ideológicas e imaginárias, de modo que a compreensão dos discursos das pessoas envolvidas no processo educativo possa ser desvelada, dentro daquele contexto em determinado momento histórico ${ }^{(1)}$.
Quanto aos aspectos éticos do estudo, de acordo com os preceitos da Resolução CNS 196/96, foi solicitada aprovação pelos: Comitê de Ética da Universidade Federal de Rio Grande - protocolo CEPAS-FURG n. 17/2009 e Núcleo de Estudos em Educação Permanente em Saúde da Secretaria Municipal de Saúde de Rio Grande. Utilizou-se, ainda, o termo de consentimento livre e esclarecido para cada sujeito participante ${ }^{(9)}$.

\section{RESULTADOS E DISCUSSÃO}

\section{Manutenções e deslizes na compreensão do pro- cesso educativo}

A partir da análise do material produzido, identificaram-se os discursos referentes ao processo educativo no ambiente de trabalho, diferenciando sentidos que se aproximam e se distanciam, por meio das paráfrases e das polissemias.

Com relação ao processo educativo, houve a manutenção de alguns sentidos, identificados a partir da marcação das paráfrases nos discursos dos sujeitos.

As profissionais de nível superior, médica e enfermeira, de forma semelhante e contínua nas entrevistas, expressaram não haver o desenvolvimento de um processo educativo na unidade, destacando como dificuldades para a sua ocorrência: a inexistência de sistematicidade, a dificuldade de reunir o grupo, as demandas organizada e espontânea, a falta de tempo e a falta de um espaço instituído.

Já os profissionais de nível médio e fundamental (auxiliares de enfermagem e agentes comunitários de saúde) identificaram que existe o desenvolvimento de um processo educativo na forma de capacitações, principalmente como processos iniciais de preparo para o trabalho, com temáticas pré-estabelecidas, desenvolvidas em local exterior à unidade básica e ofertadas por profissionais que não fazem parte da equipe multiprofissional de saúde.

Identificou-se, ainda, que todos os profissionais acreditam ser importante, tanto para a melhoria da assistência quanto para as condições de trabalho, a sistematização de um processo educativo desenvolvido na Unidade de Saúde. Todavia, quando relatam a necessidade de que a educação faça parte de seu cotidiano de trabalho, pontuam-na na forma de capacitações, treinamentos e reciclagens, sendo que alguns membros da equipe (médica e enfermeira) são percebidos - e se percebem - como os agentes 
responsáveis pelo processo educativo, tanto da equipe quanto dos outros colegas da UBSF.

Mesmo com compreensões distintas sobre a existência de um processo educativo na UBSF, todos os profissionais referem-se a este processo como o desenvolvimento de temáticas bem pontuadas, como hipertensão arterial sistêmica, diabetes, diarreias, imunização.

Observaram-se, ainda, alguns deslizes, por meio da identificação das polissemias (rupturas na continuidade do discurso da equipe), nos sentidos produzidos por determinados sujeitos.

A enfermeira, ao mesmo tempo em que refere não existir um processo educativo na Unidade, enfatiza que, informalmente, as reuniões e os momentos coletivos constituem uma troca de experiências e busca de soluções para problemas enfrentados pela equipe. Mesmo assim, reconhece não haver sistematicidade no processo. A auxiliar de enfermagem 1, mesmo destacando as capacitações como processo educativo, também salienta que as reuniões são momentos de compartilhamento de conhecimentos, de busca e de atualização.

Passado do texto para o discurso, pode-se identificar o sentido produzido naquele determinado momento e local, e ir em busca das formações discursivas que alicerçam o discurso destes profissionais de saúde.

\section{Pressupostos que materializam o conceito de educação}

A partir da identificação do mesmo e diferente, apresentado anteriormente, foi possível identificar as formações discursivas constituintes do universo de sentidos produzidos pelos profissionais de saúde daquela UBSF.

Identificar as formações discursivas (FD) que povoam o discurso dos sujeitos, em dado momento e situação da realidade na qual estão inseridos, é passo fundamental para o analista de discurso. Através das FD torna-se possível a compreensão do processo de produção de sentidos. A FD pode ser definida como aquilo que, a partir de uma posição específica em uma conjuntura sócio-histórica específica, determina o que pode e deve ser dito. Sendo assim, pode-se perceber que as palavras não possuem sentidos nelas mesmas, pois os sentidos são derivados das formações discursivas em que se inscrevem, visto que os sentidos não são predeterminados pela língua; eles se formam das relações nas e pelas $\mathrm{FD}^{(1)}$.

Os dados remeteram à formação discursiva referente a um modelo de educação tradicional, bancário, baseado na transmissão de informações, no depósito de conhecimentos, na verticalização do saber. Os profissionais referiram-se à educação como um ato exterior a eles, de ocorrência pontual, de cunho individual, destacando-se estas características em todas as entrevistas. Este resultado não corresponde à Política de Educação Permanente em Saúde, que propõe a construção de processos de educação significativos para os trabalhadores, de modo que o processo tenha, ao mesmo tempo, professores e aprendizes interagindo

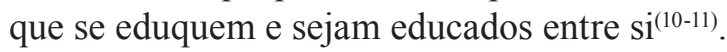

Alguns estudos recentes corroboram a premissa de que as ações educativas têm sido organizadas de forma programática e verticalizada, destacandose campanhas ou programas de saúde pública como temas destas atividades ${ }^{(12)}$.

Entende-se o desejo de atualização e de capacitação dos profissionais, visto que as mudanças e progressos tecnológicos na área da saúde são constantes, porém os processos educativos não podem se resumir a solucionar os problemas técnicos imediatos, sendo também necessária a contribuição pedagógica nas relações interpessoais, na dinâmica coletiva do trabalho ${ }^{(13)}$. A educação é uma estratégia para que os indivíduos tenham maior capacitação e é também uma possibilidade dos profissionais construírem-se dentro do mundo do trabalho, como sujeitos que constroem e desconstroem, em um movimento dinâmico e complexo mediado, por valores políticos, culturais e éticos ${ }^{(14)}$.

Mesmo com o predomínio de uma concepção de educação conservadora, incipiente e ingênua, denotou-se a FD de uma educação emancipatória, sendo destacadas as reuniões e os momentos informais como espaços de compartilhamento e construção de um novo modo dos envolvidos se entenderem como sujeitos, iguais e importantes uns para os outros e coresponsáveis pela organização e resultados das ações desempenhadas.

A educação emancipatória apresenta sujeitos em situação de protagonistas no aprendizado, em relação de horizontalidade na comunicação. Portanto, o processo educativo - entendendo-se os conceitos de homem, mundo, diálogo - vai além do ambiente formal instituído. A educação se dá em todos os lugares. Onde há gente 'existindo', há diálogo, pronúncia de mundo, desvelamento de realidades ${ }^{(8,15)}$. Estes dados apontam na mesma direção de um estudo na cidade de 
São Paulo ${ }^{(11)}$, ao afirmar que o entendimento de ações educativas como sendo reuniões entre os trabalhadores já assinala avanços na construção de espaços de troca, negociação e busca de consensos.

A instituição de uma relação dialógica no interior das unidades de saúde pode permitir a superação de relações hierarquizadas, nas quais os profissionais raramente conhecem as potencialidades uns dos outros, o que reproduz, mais uma vez, a divisão social do trabalho e estabelece relações de mando e autoridade $^{(16)}$.

Através do trabalho em equipe possibilita-se a construção de uma visão mais global e coletiva do trabalho, reforça-se o compartilhamento de tarefas e a necessidade de cooperação para alcançar objetivos comuns, de modo a dar respostas concretas às problemáticas sociais complexas. Isso permite um constante movimento teórico-prático, de forma que trabalha métodos e dispositivos novos e alternativos. Se não houver interação entre os profissionais das equipes de Saúde da Família, corre-se o risco de repetir a prática fragmentada, desumana e centrada no enfoque biológico individual, com menos valoração social dos diversos trabalhos. Isto impede o direcionamento para além de modelos de trabalho já dados e dominantes e a configuração de espaços de mudança nos processos de trabalho em saúde ${ }^{(11,16-17)}$.

Os dados deste estudo permitiram identificar formações discursivas que caracterizam dois tipos de educação, que possuem por bases conceitos e princípios distintos acerca de quais são os objetivos do processo educativo, de qual é o papel dos sujeitos e de como estes se posicionam diante da realidade. Esse processo contínuo de análise permitiu, finalmente, alcançar as formações ideológicas que alicerçam as concepções de educação para este grupo, neste momento histórico.

\section{CONSIDERAÇÕES FINAIS}

De uma forma geral, a educação vem sendo discutida na Saúde da Família, compondo temáticas de inúmeras pesquisas e ensaios, de modo que existe consenso entre estudiosos sobre a importância do processo educativo no trabalho, como estratégia de qualificação da atenção e reorientação no modelo de atenção à saúde.

Neste trabalho, observando-se a Saúde da Família como uma estratégia do SUS para reorientar o modelo assistencial, identificou-se que a educação tra- dicional, segundo as falas e posturas dos profissionais ainda é uma constante. Esta é uma questão exterior aos sujeitos, que constituídos na sua formação bancária, ainda são essencialmente fruto de uma educação tradicional, enfoque este bem destacado pela utilização da análise de discurso. A metodologia permitiu não somente a identificação dos discursos presentes nesta equipe de Saúde da Família, como possibilitou a compreensão dos dizeres numa estrutura maior de sentidos, instituídos pela educação em geral, e que são utilizados na formação de profissionais da saúde.

Os discursos identificados descortinam indagações. As concepções de educação dos profissionais na Saúde da Família apontam para a mesma direção do modelo assistencial que se pretende seguir? Acreditase que tal questionamento pode ser um propulsor para que as mudanças, ainda iniciais, se concretizem, visto que alguns sentidos diferentes já constituem as falas de determinados sujeitos, sinalizando uma direção de mudança nas formas de conceber educação no trabalho em saúde.

É essencial estabelecer um momento de interagir-interferir-repensar no fazer na área da saúde. Neste caso, a Educação Permanente - processo educativo reflexivo e significativo no ambiente de trabalho - coloca profissionais de saúde na mesma posição do processo educativo problematizador de que Freire trata em suas obras ${ }^{(12,15)}$. Trata-se da posição assumida verdadeiramente pelos homens e mulheres que desejam mais do seu local de trabalho: melhorias individuais, coletivas, de bem-estar e de satisfação.

A questão não está em valorar mais uma dimensão do trabalho do que outra, em centrar a doença e o fazer em torno dos saberes técnicos de cada profissão, nem tampouco focalizar as dimensões da saúde fundamentalmente no social. Significa desenvolver habilidades que historicamente estiveram separadas, costurar os panos que foram tecidos em separado, unir saberes de uma mesma profissão e entremeá-los com os de outras, para que se possa construir novas relações de trabalho e novas conformações organizacionais de atenção à saúde. A educação permanente em saúde, entendida nessa relação entre a formação individual, a postura profissional e o fazer coletivo durante o processo de trabalho, pode vir a contribuir para avanços no cotidiano de trabalho e com isso melhorar a qualidade da atenção e possibilitar algumas mudanças no modelo assistencial.

Assim, julga-se procedente não só o comprometimento do profissional/equipe para a realização de 
um processo educativo baseado nos pressupostos da Política de Educação Permanente em Saúde, como também uma dinâmica institucional de adesão a este processo pedagógico de forma mais ampla, que envolva a formação, a gestão, a assistência e inclusive o controle social.

\section{REFERÊNCIAS}

1. Orlandi, E. Análise de discurso: princípios e procedimentos. 7a ed. Campinas: Pontes, 2007.

2. Macedo LC, Larocca LM, Nolasco MMC, Mazza VA. Análise do discurso: uma reflexão para pesquisar em saúde. Interface - Comunicação Saúde Educação; 2008, 2(26):649-57.

3. Gomes AMT. O desafio da análise de discurso: os dispositivos analíticos na construção de estudos qualitativos. Rev Enferm UERJ. 2006;14(4):620-6.

4. Brasil. Portaria n. 648, de 28 de março de 2006. Aprova a Política Nacional de Atenção Básica (2006). [Internet] [acesso em 12 jul 2007]. Disponível: http:// tiny.cc/n8mbo.

5. Goulart FAA. Experiências em saúde da família: cada caso é um caso? [tese]. Rio de Janeiro (RJ): Escola Nacional de Saúde Pública; 2002.

6. Ceccim RB. Educação permanente em saúde: desafio ambicioso e necessário. Interface - Comunicação Saúde Educação. 2004;9(16):161-7.

7. Ribeiro ECO, Motta JIJ. Educação permanente como estratégia na reorganização dos serviços de saúde (1996). [Internet] [acesso em 10 out 2008]. Disponível: http://tiny.cc/4ffe4

8. Freire P. Pedagogia da autonomia: saberes necessários à prática educativa. $29^{\mathrm{a}}$ ed. São Paulo: Paz e Terra, 2004.

9. Ministério da Saúde (BR). Conselho Nacional de Saúde. Diretrizes e normas regulamentadoras de pesquisas envolvendo seres humanos. Resolução $\mathrm{n}$. 196, de 10 de outubro de 1996. Brasília; 1996.

10. Brasil. Portaria GM/MS n. 1.996, de 20 de agosto de 2007. Dispõe sobre as diretrizes para a implementação da Política Nacional de Educação Permanente em Saúde e dá outras providências. Diário Oficial da República Federativa do Brasil. Brasília, 22 ago 2007. Seção 1:162.

11. Peduzzi M, Del Guerra DA, Braga CP, Lucena FS,
Silva JAM. Atividades educativas de trabalhadores na atenção primária: concepções de educação permanente e de educação continuada em saúde presentes no cotidiano de Unidades Básicas de Saúde em São Paulo. Interface - Comunicação Saúde Educação. 2009;13(30):121-34.

12. Freire P. Pedagogia do oprimido. $21^{\mathrm{a}}$ ed. Rio de Janeiro: Paz e Terra. 1993.

13. Silva JAM, Ogata MN, Machado MLT. Capacitação dos trabalhadores de saúde na atenção básica: impactos e perspectivas. Rev Eletron Enferm. [Internet] 2007; 9(2) [acesso em 24 jul 2009]. Disponível: http://tiny. cc/bncdq

14. Ricaldone CAC, Sena RR. Educação permanente: uma ferramenta para pensar e agir no trabalho de enfermagem. Rev Latino-Am Enfermagem. [Internet] 2006;14(6) [acesso em 12 jul 2007]. Disponível: http:// tiny.cc/e7hiq

15. Freire P. Educação como prática de liberdade. $20^{\mathrm{a}} \mathrm{ed}$. Rio de Janeiro: Paz e Terra. 1991.

16. Araújo MBS, Rocha PM. Trabalho em equipe: um desafio para a consolidação da estratégia de saúde da família. Ciênc Saúde Colet. 2007;12(2):455-64.

17. Backes VMS. Desafios na pesquisa em enfermagem no âmbito brasileiro. Cogitare Enferm. 2009;14(4):607-11. 